REGARDS

SUR LEECONOMIE ALLEMANDE

BULLETIN ECONOMIQUE DU CRAC

\section{Regards sur l'économie allemande}

Bulletin économique du CIRAC

$85 \mid 2008$

Varia

\title{
Marché postal allemand : libéralisation sans concurrence
}

Isabelle Bourgeois

\section{CpenEdition}

\section{Journals}

Édition électronique

URL : http://journals.openedition.org/rea/492

DOI : $10.4000 /$ rea. 492

ISBN : 978-2-8218-0866-9

ISSN : 1965-0787

Éditeur

CIRAC

Édition imprimée

Date de publication : 1 mars 2008

Pagination : 5-20

ISSN : 1156-8992

\section{Référence électronique}

Isabelle Bourgeois, " Marché postal allemand : libéralisation sans concurrence », Regards sur

l'économie allemande [En ligne], 85 | mars 2008, mis en ligne le 01 mars 2010, consulté le 30 avril 2019.

URL : http://journals.openedition.org/rea/492 ; DOI : 10.4000/rea.492 


\title{
Marché postal allemand : libéralisation sans concurrence
}

\author{
Isabelle Bourgeois
}

Depuis le $1^{\text {er }}$ janvier 2008, le marché postal allemand est entièrement ouvert à la concurrence : à cette date ont pris fin le monopole de Deutsche Post AG dans le segment de la lettre de moins de $50 \mathrm{~g}$ ("secteur réservé "), de même que le régime de licence pour les sociétés opérant dans celui des envois allant jusqu'à 1000 g. La loi Postgesetz de 1997, adoptée en application de la Directive Postale 97/67/CE et entrée en vigueur le $1^{\text {er }}$ janvier 1998, s'applique désormais pleinement. Elle soumettait au principe de la liberté d'entreprise et au droit commun de la concurrence l'ensemble du marché des services postaux, à l'exception de ces deux segments qui faisaient l'objet, à titre transitoire, d'une régulation sectorielle spécifique, exercée par l'Agence fédérale des réseaux (Bundesnetzagentur, BNetzA)

En théorie, donc, le marché de la lettre est dorénavant soumis aux seules règles du marché. Or dans la réalité, le monopole de fait de Deutsche Post AG se trouve prorogé d'un côté par l'exonération de la TVA, une exception reposant en partie sur le flou juridique entourant le concept de " service universel ", et de l'autre, par l'institution d'un salaire minimum légal (jusqu'à 9,80€ de l'heure !) s'appliquant depuis le $1^{\text {er }}$ janvier 2008 à tous ses concurrents - sur un marché dont le pivot est la compétitivité-prix. Par glissements successifs dans l'interprétation des textes nationaux et communautaires, de même que sous l'effet de "relations sociales en plein bouleversement » (voir REA 83/07), à la levée des entorses réglementaires transitoires au droit de la concurrence a succédé une re-régulation immédiate via un instrument inédit : la fixation des salaires. Non seulement celle-ci crée des entraves à la concurrence d'un nouvel ordre qui défient un droit des cartels largement incompétent en matière de régulation sociale, mais elle révèle aussi à quel point l'organisation même des relations du travail est aujourd'hui menacée par un monde syndical (ver.di, en l'occurrence) qui, en optant pour une fonction tribunicienne, se dépossède de sa responsabilité d'acteur institutionnel de la régulation sociale. Cette tendance affecte particulièrement les services d'intérêt général - et se cristallise aujourd'hui sur cet ancien service public en voie de libéralisation qu'est le secteur postal.

Avec un chiffre d'affaires total estimé à 24 milliards $€$ en 2007 et plus de 400000 salariés, le marché postal allemand est le plus important d'Europe (BNetzA, 2007). II se composait jusqu'à la fin 2007 de deux segments distincts. Le premier, qui représente quelque $80 \%$ du marché, est ouvert à la concurrence depuis longtemps : il inclut la branche colis, express et logistique, de loin la plus grosse avec un CA de près de 14 milliards $€$, de même qu'une large partie des activités lettre au-delà de $1000 \mathrm{~g}$. Hautement concurrentiel, ce domaine est dominé par sept groupes: DHL (filiale de Deutsche Post AG), DPD, FedEx, GLS, Hermes Logistik, TNT (filiale de la poste néerlandaise) et UPS. Dans ce segment, et bien que les trois quarts de son chiffre d'affaires soient réalisés sur le territoire national, l'Allemagne mène nettement au sein de l'UE, avec $26 \%$ du marché européen, suivie par la France et le Royaume Uni (16\% chacun) et l'Espagne (13\%). Il est vrai que ces activités sont tirées outre-Rhin par une forte hausse de la demande de prestations orientées sur le suivi des envois (notamment par les technologies RFID) et de leur ponctualité, demande émanant des entreprises comme des particuliers. C'est là l'effet de la généralisation des process de production just in

L'Allemagne est le premier marché postal d'Europe 
La lettre, segment libéralisé au 01-01-2008, pèse 10 milliards $€$

time, mais aussi de l'essor de la VPC, dopée notamment par le B2C, qui génère aujourd'hui $32 \%$ du CA de ce segment. Si la Deutsche Post AG (DPAG) domine encore $50 \%$ du marché du colis $\mathrm{B} 2 \mathrm{C}$, elle a trouvé un sérieux challenger dans le groupe Hermes Logistik (prestataire attitré du vépéciste Otto) qui disposait à la fin 2007 d'un réseau de 13562 points de collecte, soit plus que les 12628 guichets de DPAG. Dans ce segment, la concurrence ne s'effectue pas seulement sur la qualité (et/ou la valeur ajoutée) des prestations, mais aussi et avant tout sur la taille des réseaux, comme le révèle l'annonce faite par l'opérateur historique de porter à 13500 le nombre de ses «postpoints » au début 2008.

Le second segment - pour simplifier : le marché de la lettre - se partage à égalité entre un "secteur réservé » (ex-monopole de DPAG) et un secteur soumis à licence (5 milliards $€$ de CA chacun). C'est ce segment-là qui s'est ouvert à la concurrence le $1^{\mathrm{er}}$ janvier 2008, et c'est sur lui que se concentrent les enjeux et débats.

En comparaison européenne, il semble moins dynamique : le nombre d'envois par habitant n'atteint que 259 par an, soit moins qu'en France (294) ou encore moins qu'en Finlande, championne européenne avec 521 envois (BNetzA, 2006). Certes, les Allemands recourent moins au chèque pour leurs paiements que les Français et se sont appropriés plus tôt les modes de communication électroniques. Mais en Allemagne, bien qu'elle ait légèrement baissé (-5\%) depuis 2002, la tarification reste élevée en comparaison européenne : l'affranchissement de la lettre standard (<20 g, monopole) se montait à $0,55 €$ en novembre 2007 ; s'il atteignait $0,54 €$ en France, il tombait à $0,34 €$ au Royaume Uni (BNetzA, 2007). L'ouverture totale au marché pourrait redynamiser la lettre en en abaissant le prix, mais aussi en diversifiant la qualité des prestations proposées.

Dorénavant, les Allemands (entreprises et particuliers) ont trois possibilités pour effectuer leurs envois, quel qu'en soit le poids. Soit ils les postent à un guichet de DPAG, soit ils les confient à un prestataire qui les achemine vers le réseau de DPAG ou les y collecte, soit encore, ils contractent avec un opérateur de réseau concurrent, comme PIN Group AG (qui appartient majoritairement à des éditeurs de presse) ou TNT Post AG (autre filiale de la poste néerlandaise), pour ne citer que les deux principaux challengers de l'opérateur historique. Or c'est entre ces trois opérateurs de réseau que se jouera pour l'essentiel la concurrence qui s'engage officiellement depuis le $1^{\mathrm{er}}$ janvier 2008.

\section{Le marché de la lettre commence à se structurer}

Deutsche Post AG en position de quasi-monopole

Faible dynamique sur le marché concurrentiel de la lettre

Une rentabilité encore faible
Si DPAG se taille la part du lion sur l'ensemble du marché des services postaux (les deux tiers), l'opérateur reste en position de quasi-monopole sur le segment anciennement réglementé, avec quelque $90 \%$ en volume des envois comme en chiffre d'affaires. Au secteur réservé (« licence légale exclusive »), il faut en effet ajouter les parts de l'opérateur historique dans les activités soumises au régime de licence (envois d'un poids inférieur ou égal à $1000 \mathrm{~g}$ ) : à savoir $69 \%$ des envois et 79,4 \% du CA en 2006 (BNetzA, 2006).

En dix ans, ce dernier segment n'a que peu évolué, bien que son périmètre ait été progressivement élargi au fil de la révision de la directive européenne sur les services postaux de 1997 et de l'adaptation du droit allemand. Les seuils définissant le secteur réservé ont en effet été abaissés, passant de $200 \mathrm{~g}$ maximum pour une lettre affranchie à un montant limité à cinq fois le tarif de base en 1998 à $100 \mathrm{~g}$ et trois fois le tarif de base en 2003 (en application de la nouvelle directive 2002/39/CE), puis à $50 \mathrm{~g}$ et deux fois et demie le tarif de la lettre standard en 2006 (soit 1,375 €). Malgré cet élargissement du marché pour les nouveaux entrants, et malgré une progression constante de leurs activités, le CA des 2245 entreprises sous licence recensées en 2006 ne devrait avoir atteint que 1,27 milliard $€$ en 2007, estime la Bundesnetzagentur (rapport 2007).

Ce secteur très intensif en main d'œuvre, peu rentable encore (la moitié seulement des entreprises étaient bénéficiaires en 2006) et où exercent essentielle- 
ment des PME de moins de 50 salariés et au CA inférieur à 1 million $€$, présente un faible degré de concentration, caractéristique de tout marché émergent. La réglementation contribue par ailleurs à expliquer que seule une vingtaine d'entreprises ait pu réaliser un CA supérieur à 10 millions $€$ : le volume du courrier à traiter dans les catégories supérieures à $50 \mathrm{~g}$ est/était en effet insuffisant pour générer l'effet de taille nécessaire sur ce marché où la masse salariale constitue un coût incompressible. Sur les 17,36 milliards de lettres de moins de $1000 \mathrm{~g}$ acheminées en 2006, seul 1,58 million était ainsi à mettre à l'actif des concurrents sous licence. Seuls PIN Group AG et TNT Post AG parviennent à réaliser, ensemble, un CA de plus de 400 millions $€$ (2006). Malgré cela, leur part de marché reste résiduelle, avec $8 \%$ dans le segment des licences, mais $4 \%$ seulement sur l'ensemble du marché de la lettre (BNetzA, 2007).

Structure des entreprises sous licence par taille de CA

\begin{tabular}{|c|c|c|c|c|c|c|}
\hline CA & 1998 & 2000 & 2002 & 2004 & 2006 & $2007^{a}$ ) \\
\hline$\leq 10000 €$ & 30 & 91 & 96 & 181 & 116 & 110 \\
\hline $10001 €-100000 €$ & 51 & 178 & 186 & 263 & 190 & 197 \\
\hline $100001 €-500000 €$ & 26 & 129 & 149 & 175 & 108 & 110 \\
\hline $500001-1$ million $€$ & 3 & 23 & 32 & 53 & 39 & 43 \\
\hline$>1$ million - 10 millions $€$ & 7 & 15 & 41 & 77 & 103 & 119 \\
\hline$>10$ millions $€$ & 3 & 4 & 7 & 10 & 21 & $23^{b)}$ \\
\hline
\end{tabular}

Source : Bundesnetzagentur, Tätigkeitsbericht 2006/20007. a) estimations des entreprises; b) ce groupe comprend 4 entreprises au CA supérieur à 50 millions $€$.

Structure des entreprises sous licence par taille d'effectif

\begin{tabular}{|crrrrrr|}
\hline Nombre de salariés & $\mathbf{2 0 0 0}$ & $\mathbf{2 0 0 1}$ & $\mathbf{2 0 0 2}$ & $\mathbf{2 0 0 3}$ & $\mathbf{2 0 0 4}$ & $\mathbf{2 0 0 5}$ \\
$\mathbf{0}-\mathbf{1}$ & 52 & 70 & 98 & 148 & 109 & 114 \\
$\mathbf{2}-\mathbf{5}$ & 89 & 95 & 119 & 123 & 153 & 182 \\
$\mathbf{6}-\mathbf{1 0}$ & 62 & 59 & 73 & 80 & 92 & 95 \\
$\mathbf{1 1} \mathbf{- 5 0}$ & 172 & 185 & 167 & 186 & 204 & 184 \\
$\mathbf{5 1 - 1 0 0}$ & 17 & 30 & 30 & 48 & 56 & 72 \\
$\mathbf{1 0 1 - 5 0 0}$ & 17 & 25 & 23 & 29 & 45 & 53 \\
$\mathbf{>} \mathbf{5 0 0}$ & 4 & 6 & 5 & 12 & 12 & 16 \\
Total & 413 & 470 & 515 & 626 & 671 & 716 \\
\hline
\end{tabular}

Source : Bundesnetzagentur, 10. Marktuntersuchung für den Bereich der lizenzpflichtigen Postdienstleistungen, 2006.

Après la phase initiale de création d'entreprises (filiales d'intégrateurs étrangers, start ups allemandes), se dessine depuis 2002 une structuration du marché. Elle se traduit d'un côté par une tendance au grossissement de la taille des sociétés. Une enquête effectuée à l'été 2007 auprès de 1030 entreprises sous licence par la Bundesnetzagentur (2006) révèle également l'amélioration de la rentabilité qui en découle : $68 \%$ des entreprises au CA supérieur à $500000 €$ sont aujourd'hui bénéficiaires. II s'agit dans l'ensemble d'entreprises plus âgées, donc plus expérimentées et qui ont accrû leurs investissements dans l'automatisation en prévision de l'ouverture totale du marché postal. Le panel de l'enquête comprenait 900 concurrents de DPAG, c'est-à-dire ceux parmi les 2245 détenteurs de licence qui en font réellement une exploitation commerciale. Un an plus tard, le nombre de ces « concurrents actifs » était tombé à 750 (BNetzA, 2007).

Cette structuration se traduit aussi par une accélération de la concentration, très nette à partir de 2006, année où une centaine d'entreprises s'est retirée du marché - non plus pour insolvabilité comme ce fut le cas au début de la libéralisation, mais du fait de fusions-absorptions. En 2006 et 2007, les sociétés PIN Group AG et TNT Post ont ainsi acquis à elles deux 164 PME licenciées (BuNetzA, 2007). En prévision de l'ouverture du marché, les détenteurs d'une licence ont par ailleurs déployé diverses stratégies de développement, parmi lesquelles la prise de participations : 84 entreprises étaient ainsi entrées au capital de sociétés oeuvrant dans la même branche (BNetzA, 2006).

Plus significatif encore de cette industrie de réseau est le renforcement de la coopération, sur laquelle les nouveaux entrants avaient misé très tôt, dès 1999,
Un marché en structuration accélérée depuis 2002

Concentration des acteurs... ... et, plus encore : coopération 
Montée en puissance de la sous-traitance pour mutualiser les investissements (matériel), optimiser leurs infrastructures et générer un effet de volume indispensable à leur rentabilité. Bien qu'elles soient ainsi liées économiquement et commercialement, les sociétés préservent leur indépendance juridique: elles restent des personnes morales indépendantes. Quelque 300 détenteurs de licence travaillent ainsi en partenariat, ce qui élargit considérablement leur rayon d'action et leur permet de couvrir l'ensemble du territoire - potentiellement du moins, puisque jusqu'ici, ces entreprises ne pouvaient coopérer que si elles disposaient du même type de licence, lié à une prestation spécifique.

Enfin, la sous-traitance s'est largement développée elle aussi. L'autorité de régulation a recensé 151 sociétés licenciées ayant ce type d'activités, dont 30 qui les exercent presque exclusivement (à $90 \%$ ) et 43 qui réalisent de la sorte plus de la moitié de leurs affaires. Par ailleurs, 18 sociétés travaillent notamment comme sous-traitants de DPAG (2006). Certaines d'entre elles ont été créées par externalisation dans le fil de la rationalisation de DPAG.

\section{PIN Group AG}

Cette société est née en 2005 à Berlin de la coopération des éditeurs de presse Axel Springer, Georg von Holtzbrinck et WAZ qui ont mis en commun notamment leurs réseaux de distribution. Conjointement avec la société Rosalia AG (Luxembourg), ce consortium s'est doté d'une holding, sise dans le Grand Duché : la PIN Group AG, en charge du développement stratégique des activités, le réseau des filiales allemandes en assurant la gestion opérationnelle. Ces filiales desservent des marchés locaux et régionaux. II s'agit principalement de PIN AG (Berlin, Cologne, Francfort/Main et Leipzig), de WPS (conurbation de la Ruhr), de ThPS (Thuringe) et de PDV (Hambourg). Le partenariat permet ainsi de couvrir l'ensemble du territoire allemand, d'autant que le groupe n'a cessé depuis d'en combler les lacunes grâce au rachat de divers prestataires, dont BNS (sud-ouest de l'Allemagne), OstseePost et LN Briefkurier (au bord de la Baltique) ou HNA Postlogistik et PSW Postservice Westalen (nord de la Hesse et Westphalie). Le transit entre ces différents réseaux a été confié en avril 2007 à un groupement de transporteurs, la société XAnto.

PIN Groupe AG vise dans un premier temps la clientèle des entreprises pour y réaliser les effets de taille indispensables à la conquête du segment des particuliers. Dans ce cadre, il a engagé des négociations avec le réseau de filiales des Caisses d'épargne allemandes (Sparkassen) afin de porter à moyen terme le nombre de ses points de vente à 12000 .

Le groupe, qui occupe plus de 9000 salariés dans 91 filiales, déclare un volume quotidien d'envois de 3,2 millions de plis. S'il a réalisé au cours des neuf premiers mois de 2007 un CA de 195,3 millions $€$, ses activités sont encore largement déficitaires (47,8 millions $€$ ). Et il a engagé une procédure d'insolvabilité (redressement) en décembre 2007.

Sources : Bundesnetzagentur (2007), www.pin-group.net

\section{TNT Post AG \& Co. KG}

TNT Post AG \& Co. KG (Hanovre) est un joint venture entre l'opérateur postal néerlandais TNT Group $(71 \%)$ et le groupe allemand de logistique Hermes $(29 \%$, Hambourg, filiale du vépéciste Otto). II dessert l'ensemble du marché allemand depuis 2000. Ses nouvelles activités lettre s'insèrent dans un ensemble plus large, la poste néerlandaise étant un acteur majeur sur le marché du courrier de l'express et de la logistique, notamment grâce à un réseau de coopération mondial.

TNT Post est pour l'instant spécialisé dans la clientèle des entreprises à laquelle elle offre l'ensemble de la gamme des prestations postales, mais se prépare lui aussi à développer ses activités à destination des particuliers.

Depuis 2005, le groupe a étendu son réseau en créant 4000 succursales pour la distribution (notamment dans les régions à forte concentration d'entreprises : Rhénanie du Nord-Westphalie, Hambourg, Francfort/Main et Stuttgart) ou en entrant au capital de plus de 150 distributeurs. Deux participations sont stratégiques : Nordwest-Mail GMBH (Brême ; $25,1 \%$ ) dont le réseau CITIPOST dessert tout le nord-ouest de l'Allemagne (et couvre 400000 foyers à Brême) et ecoflash (Munich, 25,1\%, à l'origine un joint venture entre les groupes d'édition Ippen et Süddeutscher Verlag), ce qui assure à TNT Post la couverture de 1,6 million de foyers dans l'agglomération de Munich. TNT Post vient également de racheter la société PostCON, le principal gestionnaire de centres de tri d'Allemagne (13 sites) après DPAG. Si on y ajoute le réseau de Hermes $(90 \%$ des foyers allemands), TNT Post couvre aujourd'hui la totalité du territoire allemand et prévoit la création de 13500 points de vente

TNT Post (volume quotidien de publicité non adressée : 40 millions d'unités) réalise un CA de 200 millions $€$ (2006) et affirme avoir investi 100 millions $€$ dans l'extension de son réseau Lettre. Il occupe plus de 6000 salariés, dont 4000 coursiers.

Sources : Bundesnetzagentur (2007), www.tnt.de
Un maillage étroit des réseaux sur tout le territoire

Le marché se structurera autour des intégrateurs
Si la structure des entreprises reste pour l'instant hétéroclite, les diverses stratégies de rapprochement poursuivies ont donné naissance, en une décennie, à un maillage étroit de l'ensemble du territoire allemand en termes de réseau de collecte et de distribution. Certes, ce réseau n'est pas d'un seul tenant ni unique, mais il couvre l'ensemble des segments des prestations de logistique. Virtuellement, il crée les mêmes conditions de base (accessibilité et proximité) que le réseau historique de DPAG. Cela vaut tout particulièrement pour les réseaux de distribution des éditeurs de presse. Le fait que, outre-Rhin, près des deux tiers de la diffusion des quotidiens par exemple se fait par abonnement (portage à domicile) donne une idée de la densité et de l'efficience de ces réseaux.

Comme dans les autres industries de réseaux en voie de libéralisation, par exemple les télécommunications (voir REA 78/06), les modèles économiques des 
nouveaux acteurs se diversifient selon les segments considérés. A l'heure actuelle, seuls PIN Group AG et TNT Post se sont hissés au rang d'intégrateur, disposant de leurs propres infrastructures et offrant l'ensemble de la chaîne des services postaux auxquels va s'adjoindre la lettre. C'est au niveau des intégrateurs que devrait donc s'installer le marché concurrentiel, estime la Bundesnetzagentur (2007), surtout dans les régions à forte densité de population - autant dire sur une très large partie du territoire allemand, l'Allemagne ne comptant que peu de zones à faible densité en comparaison de la France.

Pour un certain nombre d'autres prestataires, spécialisés dans la collecte d'envois auprès de l'expéditeur ou de la distribution vers le destinataire, s'est posée, à l'image des télécommunications ou de l'électricité, l'épineuse question de l'accès des tiers au réseau - à celui de DPAG, en l'occurrence. L'accès n'est possible à grande échelle que depuis une décision prise en février 2005 par l'Office fédéral des Cartels, mais reste peu rentable pour deux raisons : ces prestations sont soumises à la TVA (ce qui accroît leur prix au consommateur), à l'inverse de celles de DPAG, exonérées, ce qui permet à l'opérateur historique de négocier des tarifs plus avantageux avec ses clients, rappellent la Bundesnetzagentur, de même que la Commission des monopoles (rapports 2007). Ensuite, la tarification de l'accès était réglementée en amont (ex ante), la DPAG justifiant son prix élevé par ses obligations de service universel comme les lourdes charges (sociales) liées à sa désétatisation et sa privatisation partielle. Enfin, un grand nombre de ces prestataires s'est plaint des pratiques commerciales de DPAG qui refusait les envois au motif qu'ils étaient mal triés ou que les adresses n'étaient pas conformes. Pourtant, si on fait abstraction de ces limitations et désagréments, les activités de ces entreprises au rayon d'action avant tout local ou régional sont potentiellement porteuses car elles offrent une complémentarité avec celles des intégrateurs, notamment dans les régions à faible densité de population où elles permettront d'offrir un service accessible à tous et à un prix abordable. Dans cette catégorie aussi, la concurrence pourrait porter sur les réseaux et forcer la coopération entre prestataires locaux et régionaux pour atteindre une taille critique.

Plus généralement, la diversification des business modèles comme l'innovation en matière de services sont restées quelque peu bridées par un régime de licences contraignant et, surtout, standardisant. Celui-ci, qui reposait sur deux critères : le type de prestations et la taille du marché desservi, imposait en effet des prestations soit complémentaires aux services offerts par DPAG, soit à valeur ajoutée, mais toujours définies selon l'offre traditionnelle. Les catégories de licences étaient au nombre de huit, classées de $\mathrm{A}$ à $\mathrm{H}$ (et déclinées selon les différents échelons territoriaux ; voir REA 65/04).

Les licences de loin les plus prisées par les nouveaux entrants étaient celles des services «de qualité supérieure » se différenciant des prestations de service universel par leur valeur ajoutée (ex-catégorie D). Elles seules permettaient en effet d'innover réellement : distribution le jour même, du soir au lendemain matin, ou traçabilité du courrier. Alors qu'en 2000, la part de marché de cette catégorie n'atteignait que $27 \%$ du total du CA du segment sous licences, elle a dépassé les $50 \%$ en 2005 pour se situer à $56 \%$ en 2007 (BNetzA, 2007) - malgré un risque procédurier prononcé. La forte croissance de ces activités reflète la demande accrue des entreprises, tirée, comme le rappelle la Commission des monopoles, par une tarification inférieure à celle de DPAG $(0,52 €$ pour une lettre standard au lieu de $0,55 €$ ) pour une qualité de service, au niveau des délais, nettement supérieure à celle imposée par les standards de service universel.

Le décret sur le service universel postal (Post-Universaldienstverordnung) en vigueur jusqu'au 31-12-2007 prévoyait $80 \%$ de J+1 ou $95 \%$ de J+2 (jours ouvrables) pour le courrier intra-allemand. La DPAG qui s'enorgueillit de respecter ces délais prend pour critère la durée de transit du courrier au sein de son réseau après la levée des boîtes aux lettres (la dernière s'effectuant généralement à 17 heures). Or pour le consommateur que ce décret était censé protéger, comme le rappelle la Commission des monopoles, la durée d'acheminement se calcule à partir du
L'accès des tiers au réseau de DPAG a longtemps été difficile

Des services encore peu innovants du fait de la conception même des catégories de licences

Une nette prédilection pour les services de qualité supérieure...

... bien que DPAG ait tenté d'entraver leur essor 

la demande des entreprises

Effet positif des licences sur les régions en restructuration
Elément clef du marché :

moment où le pli est glissé dans la boîte aux lettres. Certes, selon les données qu'a pu recueillir l'autorité de régulation, les attentes des consommateurs sont dans l'ensemble satisfaites par DPAG, du moins ces deux dernières années. Mais ce qui a posé problème pendant près de dix ans, c'est la définition de la valeur ajoutée des services de licence $D$, se traduisant par une intense activité procédurière de DPAG (voir REA 65/04) qui redoutait cette concurrence dans son cœur d'activité. Ce n'est qu'en juin 2007 qu'une série d'arrêts du Tribunal administratif fédéral a précisé cette notion, mettant fin aux litiges. Désormais, la valeur ajoutée du J+1 proposé par les concurrents de DPAG réside sans conteste dans le fait que le courrier est collecté après 17 heures chez le client et livré au destinataire le lendemain (jour ouvrable) avant midi. Ce service-là aussi, fort prisé des entreprises et administrations à l'échelon local/régional, a un fort potentiel de développement.

Des partenariats devraient s'engager avec les détenteurs des ex-licences $C$ et $G$ (courrier inter-entreprises et courrier transfrontières sortant), dans la mesure où leurs prestations s'adressent majoritairement aux entreprises et que ces dernières génèrent $90 \%$ de la demande sur le marché postal. II n'est pas étonnant dès lors que ce segment d'activités à haut potentiel présente le plus fort taux de concentration. Une diversification par type d'activités est donc prévisible, les offreurs de services se concentrant sur la clientèle des entreprises, les opérateurs de réseaux investissant le marché de masse (lettre) des particuliers. Ce partage sommaire n'exclut pas pour certains, loin s'en faut, l'intégration de l'ensemble des activités du marché postal et de la logistique ni un découpage territorial des activités par taille de marché - de l'échelon local au marché mondial.

Le second critère auquel obéissait le régime des licences était la taille du marché desservi. L'instance de régulation en avait ainsi attribué 856 pour le marché national, 674 pour les marchés régionaux et 715 pour le niveau local. Ce découpage avait permis à un certain nombre de Länder et, en leur sein, de régions à faible dynamique économique, en restructuration et à haut niveau de chômage, de développer de nouveaux secteurs d'activité. C'est ainsi que le nombre de licences par million d'habitants est particulièrement élevé à l'est, de même que dans les régions les moins dynamiques de l'ouest, souvent à dominante rurale, comme le nord-est de la Bavière ou l'est de la Basse-Saxe, par exemple. Pour le dire autrement : les emplois générés par les services postaux sous licence ne se situent que marginalement dans les grandes agglomérations ou pôles à forte concentration d'activité, mais bien au contraire principalement dans les zones défavorisées ou rurales. Le régime des licences a ainsi eu un effet positif à la fois sur la dynamique de compétitivité territoriale et sur l'emploi, surtout des moins qualifiés.

\section{La lente progression de l'idée d'une « régulation » par un salaire minimum légal}

Une inconnue : l'effet de l'ouverture du marché sur l'emploi

Depuis le début de la libéralisation du secteur postal voici dix ans, la question de son impact sur l'emploi donne lieu à débat. Or aucune donnée statistique officielle fine n'existe, puisque ces activités 'lettre' sous licence ne sont pas considérées comme une branche dans la nomenclature de Destatis. Les seuls chiffres disponibles sont dès lors des estimations fondées sur des enquêtes non représentatives, ce qui ouvre une large brèche à la polémique.

De son côté, l'autorité de régulation se contentait, lors de l'étude des dossiers de candidature pour une licence, de contrôler la faisabilité du business plan déposé et, sous l'angle social, sa conformité avec le droit. Son contrôle se limitait ainsi à la phase initiale de création ou d'autorisation de l'entreprise et ne s'intéressait qu'aux catégories d'emplois : soumis à cotisations ou non, temps plein ou temps partiel. Par la suite, elle s'est penchée à intervalles réguliers sur les fluctuations qu'elle a pu observer à l'intérieur de ces grandes catégories, mais sans aller audelà des compétences que lui conférait la Loi Postgesetz dont l'art. 6, § 3, al. 1, 3 lui fait obligation de prévenir "le risque d'une distorsion de la concurrence pou- 
vant provenir du fait que les nouveaux licenciés recourent à des conditions de travail non protégées ", donc de vérifier le respect des conventions collectives, comme celui du libre exercice d'une profession et de la liberté de contracter.

Cette «clause sociale » (Sozialklausel) avait été introduite dans la loi en 1997 après d'âpres négociations du texte dans la commission de conciliation Bundestag/Bundesrat, et sur demande du SPD (de même que des Verts et du PDS) afin de réduire les risques de «dumping social » et de «précarisation de l'emploi ». Selon les avocats d'une " protection » sociale des salariés dans les anciens services publics s'ouvrant au marché, les prémisses de la libéralisation du secteur postal (sur fond de l'ouverture, peu auparavant, du marché des télécommunications) se seraient en effet soldées par la suppression de 100000 emplois et la hausse parallèle de petits boulots au détriment de l'emploi soumis à cotisations (Säcker, 2007). Dès cette époque, l'approche de la régulation de ce secteur en transition est en quête d'un délicat équilibre entre plusieurs objectifs (Bourgeois, 2001). D'abord, il s'agit de concilier (comme pour les télécommunications et, plus tard, pour l'énergie) l'application du droit normal de la concurrence, l'objectif final, avec la garantie de la pérennité et de l'extension des infrastructures (donc aussi du service universel), ce qui implique l'adoption d'un cadre réglementaire transitoire se manifestant par une régulation sectorielle spécifique aux industries de réseaux (voir REA 63/03). A cet exercice délicat, puisqu'il s'accompagne d'entorses au droit commun le temps que s'installe la concurrence, s'ajoute le défi qui consiste à concilier des sources du droit étrangères, voire incompatibles : la loi (ouverture au marché, encadrement de ce dernier) qui relève de l'Etat (législateurs), et le contrat (conditions sociales) qui relève des partenaires sociaux, donc de la société civile.

Ce défi est décuplé par la privatisation des statuts qu'impliquent la désétatisation, puis la libéralisation, notamment en termes de droit du travail, et plus important encore dans le cas allemand, par la non-organisation du secteur des services libéralisés. Faute de 'syndicat de postiers privés', ce dernier échappe à ver.di en tant que 'branche' ; quant au patronat, il n'est pas, lui non plus, constitué en partenaire social unique de branche dans ce segment. C'est ainsi que, depuis dix ans, la «clause sociale » de la Loi Postgesetz, de même que l'application strictement réglementaire (contrôle en conformité) qu'en fait la Bundesnetzagentur est un des points de cristallisation des débats sur la libéralisation des marchés postaux, la gauche allemande et Ver.di cherchant à en faire l'instrument d'une " rerégulation du secteur postal par la politique salariale » (Brandt et al., 2007).

Or, à l'instar de ce qu'on peut observer dans les chemins de fer allemands (voir $R E A$ 83/07), l'affaire se complique du fait d'un émiettement syndical croissant et de la lutte d'influence qu'elle nourrit. Une Fédération patronale des services postaux (Arbeitgeberverband Postdienste : AGV Postdienste), qui regroupe une vingtaine d'entreprises adhérentes (environ 200000 salariés) et est dominée par DPAG, a été créée ad hoc en août 2007 pour négocier avec ver.di, ainsi qu'une association elle aussi ad hoc, Tarifgemeinschaft DPVKOM/CGPT, dominée par les postiers de DPAG. Ces organisations ont signé, le 29-11-2007, une convention collective portant sur un salaire minimum horaire des facteurs variant entre $8,00 €$ et 9,80 € selon les régions et s'appliquant... aux concurrents de DPAG.

Ceux-ci avaient constitué, en réaction, un second groupe de partenaires sociaux à l'automne 2007. La représentation des salariés du 'privé' est assurée par le Syndicat des nouveaux services de la lettre et de sa distribution: Gewerkschaft neue Brief- und Zustelldienste (GNBZ), créé en octobre. En face, les organisations patronales sont au nombre de trois: la Fédération des Services Colis, Express et Logistique (Bundesverband der Kurier-Express-Post-Dienste, BdKEP), la Fédération des éditeurs de presse allemands (Bundesverband Deutscher Zeitungsverleger, $B D Z V)$, toutes deux établies depuis longtemps, et une nouvelle représentation, la Fédération patronale des nouveaux services de la lettre et de sa distribution (Arbeitgeberverband der Neuen Brief- und Zustelldienste, NBZ). Cette dernière, qui compte quelque 35 adhérents dont PIN Group et TNT Post, avait été créée en septembre 2007 en contrepoids de l'AGV dominée par DPAG, ce qui amène ver.di
La « clause sociale »: point de cristallisation des défis liés à la libéralisation...

... et à une « re-régulation » par la politique salariale

Deux blocs de partenaires sociaux : l'un, dominé par DPAG...

... l'autre, dans le secteur concurrentiel 
Au cœur des débats : la « précarisation » de l'emploi

46000 nouveaux emplois créés en dix ans, surtout à l'est

à mettre en doute sa représentativité et l'a incité à refuser d'entrer en négociation avec lui pour l'adoption d'une convention salariale propre. De leur côté, BdKEP et GNBZ avaient envisagé à l'automne une convention portant le salaire minimum horaire à 7,50 € à l'ouest (6,50 € à l'est), tentant, grâce à cette contre-proposition respectueuse des revendications de Ver.di (création d'un salaire plancher de $7,50 €)$, de barrer la route à l'institution d'un salaire minimum légal via la procédure d'extension (Allgemeinverbindlichkeitserklärung). En vain.

Cette longue non-organisation du secteur de la lettre avait bien entendu, elle aussi, contribué à alimenter les rumeurs sur l'inconnue majeure : le nombre d'emplois créés ou détruits par l'ouverture au marché, de même que les pratiques salariales. Les débats qu'elles suscitent sont communs à toutes les industries de réseaux en voie de libéralisation : la désétatisation des anciens services publics étant acquise, de même que l'application du droit privé y compris au plan social, les enjeux se concentrent sur l'emploi et sa "précarisation ».

Dans son dernier rapport d'activité, la Bundesnetzagentur conclut que les entreprises sous licence ont créé plus de 46000 nouveaux emplois depuis 1998, auxquels il faudrait ajouter les emplois générés par la tendance à la hausse de la sous-traitance observée plus récemment dans ce segment, mais dont le nombre n'est pas connu. Si le nombre d'emplois à temps plein est en augmentation, reflétant la structuration en cours du secteur comme le développement d'une offre de prestations plus complexe, les petits boulots en tous genres constituent la majeure partie des emplois (les deux tiers). Et ils se concentrent dans les entreprises effectuant la distribution du courrier, principalement dans les régions à faible densité de population comme les nouveaux Länder. Une des raisons en est le faible volume du courrier à acheminer, soumis de surcroît à de fortes variations.

Répartition régionale des emplois (unités) dans le segment anciennement sous licence (hors DPAG)

\begin{tabular}{|c|c|c|c|c|c|}
\hline Land $^{\text {a) }}$ & Temps plein & Temps partiel & Activité négligeable & Minijobs $^{c)}$ en CDD & Total \\
\hline BW & 369 & 385 & 1905 & 27 & 2686 \\
\hline BS & 539 & 238 & 1908 & 44 & 2774 \\
\hline BY & 461 & 395 & 1793 & 41 & 2690 \\
\hline B & 585 & 737 & 2915 & 183 & 4420 \\
\hline BB & 214 & 445 & 1430 & 26 & 2115 \\
\hline HB & 8 & 12 & 119 & 0 & 139 \\
\hline $\mathrm{HH}^{\mathrm{b})}$ & 2335 & 851 & 150 & 3 & 3339 \\
\hline $\mathbf{H}$ & 294 & 99 & 260 & 8 & 661 \\
\hline MP & 202 & 157 & 4620 & 254 & 5233 \\
\hline NRW & 1696 & 1315 & 3911 & 75 & 6997 \\
\hline $\mathbf{R P}$ & 171 & 170 & 1507 & 10 & 1854 \\
\hline S & 136 & 10 & 667 & 40 & 853 \\
\hline sx & 764 & 386 & 3086 & 20 & 4256 \\
\hline SA & 237 & 1763 & 1644 & 31 & 3675 \\
\hline SH & 80 & 92 & 850 & 377 & 1399 \\
\hline $\mathbf{T}$ & 349 & 207 & 1793 & 710 & 3059 \\
\hline Total & 8440 & 7307 & 28558 & 1849 & 46154 \\
\hline
\end{tabular}

Source : Bundesnetzagentur, 10. Marktuntersuchung für den Bereich der lizenzpflichtigen Postdienstleistungen (2006). Etat : 2005. a) BW : BadeWurtemberg; BS : Basse-Saxe; BY : Bavière ; B : Berlin; BB : Brandebourg; HB : Brême ; HH : Hambourg ; H : Hesse ; MP : MecklembourgPoméranie; NRW : Rhénanie du Nord-Westphalie ; RP : Rhénanie-Palatinat ; S : Sarre ; SX: Saxe ; SA : Saxe-Anhalt ; SH : Schleswig-Holstein ; T: Thuringe. b) y sont comptabilisés les emplois de PDV, filiale de PIN AG, répartis sur l'ensemble du territoire allemand. b) Geringfügige Be$\mathrm{T}$ : Thuringe. b) y sont comptabilisés les emplois de PDV, filiale de PIN AG, répartis sur l'ensemble du territoire allemand. b) Geringfügige Be-
schäftigung : activité dite minime ou négligeable, échappant de ce fait aux cotisations sociales. c) Minijobs : petits boulots d'appoint, exonérés de schäftigung: activite dite minime ou négligeable, échappant
cotisations et d'impôts jusqu'à $400 €$ de salaire mensuel.

Et 20000 emplois externalisés par DPAG
A ces 46000 emplois créés par les sociétés sous licence, il faut ajouter entre 20000 et 25000 emplois (équivalent temps plein) externalisés depuis 1997 par DPAG dans le cadre de la rationalisation de sa division Lettre et en quelque sorte 'transférés' à des sociétés sous-traitantes. DPAG a ainsi externalisé les deux tiers de ses activités de transport (levée des boîtes aux lettres, acheminement vers les centres de tri, etc.), ce qui s'est traduit par le transfert de quelque 8000 emplois en équivalent temps plein à des sociétés de taxis ou des transporteurs selon les distances à couvrir. De même a été externalisée la gestion de quelque 8000 agences ou points poste, confiée à des commerçants sous contrat (supérettes, 
marchands de journaux ou stations-service). C'est ce qui ressort d'une étude commanditée par la Bundesnetzagentur à un institut de recherche scientifique (Wissenschaftliches Institut für Infrastruktur und Kommunikationsdienste, WIK) et publiée en mai 2007. DPAG ne communiquant guère sur ces réductions d'effectifs et l'autorité de régulation devant se contenter d'enquêtes auprès des détenteurs de licences, le périmètre de la sous-traitance de DPAG souffrait d'une méconnaissance à laquelle l'étude WIK remédie en partie. Elle apporte également des informations plus fines sur la structuration sociale du marché de la lettre.

L'étude repose sur une enquête menée auprès de 38 entreprises réalisant au total $45 \%$ du CA soumis au régime des licences (dont le périmètre de la licence exclusive de DPAG). Leurs réponses ont été confrontées avec les horaires, les congés et les salaires en vigueur dans des branches aux activités et qualifications comparables (imprimerie, commerce de détail, VPC, gastronomie/hôtellerie, transport routier) - à l'exclusion de celles en vigueur chez DPAG, considérées comme trop particulières.

Le WIK a suivi en cela la conclusion à laquelle était parvenu un rapport commandité par la Bundesnetzagentur au Prof. Franz. Jürgen Säcker, Directeur de l'Institut de droit de l'économie, de la concurrence et de la régulation, allemand et communautaire, de l'Université libre de Berlin. II considère en effet que l'opérateur ne peut servir de référence du fait de son statut de monopoliste et que, au contraire, pour juger des "conditions de travail usuelles " dans le segment postal qui s'ouvre à la concurrence, il convient d'étudier celles en vigueur aux différents échelons locaux dans des branches voisines. Cela étant, dans le débat qui couvait avant la libéralisation totale du marché (et qui couve toujours), DPAG a toujours représenté une référence implicite, ce qui avait amené l'institut WIK à lui consacrer une place particulière.
L'étude WIK cherche à recenser les conditions de travail usuelles chez les concurrents de DPAG

\section{Référence implicite : les conditions de travail très particulières chez Deutsche Post AG (Division Lettre)}

Les conditions de travail de l'ensemble des salariés de DPAG sont régies par la convention-cadre de septembre 2003 qui crée des conditions-cadre uniques sur l'ensemble du territoire : durée hebdomadaire du travail ( 38,5 heures sur la base d'une semaine de 5 jours) et congés (de 26 à 30 jours selon l'âge).

Dans la division Lettre, les salaires sont régis par une convention valable pour les seuls salariés de DPAG (143 200). Signée par la DGAP et les syndicats ver.di, DPVKOM et CGPT, elle est en vigueur depuis novembre 2006 et elle expire le 30-04-2008. Cette convention ne s'applique pas aux filiales Deutsche Post In Haus Service ou Deutsche Post Print/Com où les salaires sont inférieurs. Dans d'autres filiales, comme First Mail Düsseldorf GmbH, aucune convention tarifaire n'existe.

Cette 'convention-maison' (Haustarifvertrag), un type de contractualisation rare en Allemagne où prévaut le périmètre de branche et non d'entreprise, prévoit des dispositions communes à toutes les catégories de salariés : rémunération mensuelle au forfait, prime de congés de $332 €$ et $13^{\mathrm{e}}$ mois équivalent à un salaire mensuel. Elle prolonge également la coexistence de deux statuts salariaux (justifiée par la transition de cette ex-administration vers une société par actions), selon que les salariés ont été embauchés en CDI avant (garantie des droits acquis) ou après une date butoir : 01-01-2001 pour les ouvriers, 01-01-2003 pour les employés. Selon les estimations de l'institut WIK (la DPAG ne communique pas de chiffres sur ce point), les salariés se répartissent à égalité entre ces deux statuts qui se traduisent par des écarts salariaux pouvant aller jusqu'à $40 \%$ pour la même activité.

Planchers et plafonds salariaux en vigueur à DPAG (en€/heure)

\begin{tabular}{|c|c|c|c|}
\hline Activités & Salaire minimum & Maximum nouveau régime & Maximum droits acquis \\
\hline Employé tri & 9,63 & 11,69 & 11,69 \\
\hline Chauffeur courte distance & 9,63 & 11,69 & 11,69 \\
\hline Chauffeur longue distance & 10,14 & 12,72 & 12,72 \\
\hline Facteur & 10,14 & 12,72 & 12,72 \\
\hline Agent commercial & 9,63 & 12,72 & 12,72 \\
\hline Technicien commercial & 10,14 & 24,08 & 24,08 \\
\hline
\end{tabular}

L'étude WIK a donc cherché à comparer les conditions de travail en vigueur dans les divers secteurs concernés par les activités confiées par DPAG à ses soustraitants : distribution, transport et points poste. Ces secteurs, régis le plus souvent par des conventions collectives propres à la branche et conclues au niveau régional, ont tous en commun de présenter les fortes disparités, habituelles en
Sous-traitants de DPAG : les taxis ont les salaires moyens les plus bas $(6 € / \mathrm{h})$ 
Concurrents de DPAG : des minima aux alentours de $6 €$

Allemagne. Dans la branche des taxis (où la moitié des actifs a une activité 'courrier') qui n'est régie par une convention salariale qu'en Bavière, le salaire moyen est ainsi de $6 € / \mathrm{h}$. Dans celle du transport routier, la moyenne des six conventions régionales étudiées s'établit à $9,50 € / \mathrm{h}$. Dans celle du commerce de détail (une convention par Land) enfin, elle est de 8,50 €/h. Dans ces trois secteurs, la durée moyenne hebdomadaire du travail s'échelonne de 52,5 heures à 38,5 heures. Quant à la durée des congés annuels, elle va de 30 à 27 jours.

Dans le segment anciennement sous licences dans lequel opèrent les concurrents de DPAG, aucune convention n'existait au moment de l'enquête menée par l'institut WIK. Celle-ci révèle que, dans la moitié des cas, les salariés y sont rétribués au forfait (mensuel) - sauf deux catégories : les facteurs et les agents commerciaux. La moitié d'entre eux (un quart du total des salariés) a une rémunération variable sur la base d'un fixe ; c'est le cas principalement des porteurs de journaux qui complètent leur fixe en distribuant des lettres (entre 0,10 et 0,15€ par exemplaire). Enfin, le quart restant est payé exclusivement au nombre de plis acheminés. En moyenne, l'institut a calculé un salaire horaire moyen de 8,44€ dans ces nouveaux services, mais cette moyenne masque d'importantes disparités régionales. La durée hebdomadaire du travail (temps complet) varie entre 40 et 38,5 heures. En matière de congés annuels, tous les employeurs respectent les dispositions de la Loi sur les congés minima des salariés (Mindesturlaubsgesetz für Arbeitnehmer, BUrlG) : une semaine de cinq jours ouvre droit à 20 jours annuels, ce nombre passant à 24 pour une semaine de 6 jours.

Salaires minima et maxima versés par les concurrents de DPAG (en €/heure)

\begin{tabular}{|lccc|}
\hline Activités & Salaire minimum & Maximum & Moyen \\
Employé tri & 5,00 & 13,84 & 8,36 \\
Chauffeur courte distance & 6,00 & 11,00 & 7,64 \\
Chauffeur longue distance & 6,25 & 10,50 & 8,20 \\
Facteur & 5,50 & 13,00 & 7,94 \\
Agent commercial & 6,72 & 25,00 & 14,39 \\
Technicien commercial & 6,00 & 28,90 & 16,22 \\
\hline
\end{tabular}

Source des données : WIK, Arbeitsbedingungen im Briefmarkt, 2007.

Conditions de travail dans quelques branches voisines (régies par des conventions)

\begin{tabular}{|lcccc|}
\hline Branche & Heures/sem. & Congés (moyenne) & Salaire minimum & ... et maximum \\
Imprimerie & 36,5 & 30 jours & $6,03 € / \mathrm{h}$ & $23,70 € / \mathrm{h}$ \\
Commerce de détail & 37,7 & 27,7 jours & $7,92 € / \mathrm{h}$ & $17,35 € / \mathrm{h}$ \\
VPC & 37,7 & 27,7 jours & $5,41 € / \mathrm{h}$ & $23,22 € / \mathrm{h}$ \\
Gastronomie/Hôtel. & 38,9 & 27,5 jours & $4,97 € / \mathrm{h}$ & $12,17 € / \mathrm{h}$ \\
Transport routier & 40,4 & 28 jours & $7,57 € / \mathrm{h}$ & $18,14 € / \mathrm{h}$ \\
Gestion de données & $\left.34,0^{*}\right)$ & 30 jours & $5,95 € / \mathrm{h}$ & $30,41 € / \mathrm{h}$ \\
\hline
\end{tabular}

Source des données : WIK, Arbeitsbedingungen im Briefmarkt, 2007. *) Régime particulier de Deutsche Telekom.

Conclusion de l'étude WIK : des conditions de travail hors normes chez DPAG
L'étude parvient à la conclusion que, dans l'ensemble, les conditions de travail dans le marché de la lettre à la veille de son ouverture sont celles d'un secteur à bas salaires et qu'elles reflètent la spécificité d'un secteur intensif en main d'œuvre faiblement qualifiée et soumis à de fortes fluctuations en ce qui concerne les volumes à acheminer ou distribuer ; la réactivité au marché, de même que la flexibilité de la gestion des coûts requises expliquent la place accordée à une rémunération à l'unité. Mais hormis cette particularité, ce marché ne présente pas de caractéristiques exceptionnelles, les employeurs respectant la loi (congés) comme les usages de la régulation contractuelle (durée du travail). Dans ce contexte, il appert que c'est plutôt la rémunération de DPAG qui atteint " un niveau inhabituel dans la branche », de même qu'est «inhabituelle » l'existence d'une grille salariale unique valable sur l'ensemble du territoire, " y compris en comparaison avec d'autres branches ». Cela dit, aucune donnée régionale assez fine (croisant les normes locales à la fois par branches et par métiers) n'est disponible pour établir cette grille de référence pour l'évaluation des "standards de protection sociaux » évoqués par l'art. 6, § 3, al. 1, 3 de la Loi Postgesetz. 
Cette lacune, de même que l'échantillon restreint à la base de l'enquête de l'institut WIK, avaient incité l'autorité de régulation à lancer peu après, en juin 2007, sa propre enquête auprès de quelque 1500 détenteurs de licence. Mais elle s'était vu opposer un recours en référé déposé par 46 d'entre eux ; l'affaire n'ayant pas encore été jugée sur le fond, l'enquête n'a pas abouti jusqu'ici.

De son côté, le syndicat ver.di avait commandité une étude au cabinet Input Consulting, publiée en décembre 2006 sous le titre : « Libéralisation et précarisation conditions de travail chez les nouveaux prestataires de la lettre en Allemagne ». Elle est parcellaire elle aussi, reposant sur un panel de 53 entreprises, de surcroît non situées dans leur contexte (chiffre d'affaires ?), donc non représentatif. A la différence de l'étude WIK dont le panel est anonymisé, celle d'Input inclut les sociétés PIN et TNT. Comme l'étude concurrente, elle se concentre sur les facteurs ou autres salariés dans la distribution - la catégorie "la plus nombreuse et la moins bien payée en tendance »(Commission des monopoles). Et elle parvient grosso modo aux mêmes conclusions: DPAG constitue une exception, avec un salaire médian de $11,84 € / h$ (contre $11,29 € / h$ pour WIK) en comparaison de salaires médians allant de $7 € / h$ (ouest) à $5,90 €$ (est) chez les concurrents.

Les données collectées par l'étude d'Input sont aussi irréprochables que celles de WIK, de même que son analyse macro-économique qui prévoit elle aussi un essor relativement lent du marché de la lettre du fait de l'existence d'un certain nombre de barrières d'entrée résultant du monopole de DPAG. «Dans un tel marché quasi-saturé, la seule perspective qui s'offre aux nouveaux entrants est celle d'une concurrence par élimination. En Allemagne, celle-ci s'effectue jusqu'ici majoritairement via les prix ». Si une étude du cabinet PwC pour la Commission européenne estime que, d'ici 2011, la part de marché des nouveaux entrants devrait atteindre $20 \%$, rien ne permet d'affirmer comment se répartiront les parts entre PIN Group, TNT Post et les groupes postaux étrangers (La Poste, Royal Mail, poste autrichienne, suédoise, voire UPS) qui s'apprêtent à entrer sur le marché allemand, explique Input. Cela dit, la puissance de leur réseau de distribution des quotidiens présente un sérieux avantage compétitif pour les éditeurs de presse réunis dans PIN Group, que l'étude illustre d'une citation de son président Günther Thiel (démissionnaire à l'automne 2007) : «... nous pouvons offrir un tarif inférieur... car en ce qui concerne la distribution de la lettre, le segment le plus coûteux de l'ensemble du système de distribution, nous n'avons à tenir compte que des coûts complémentaires, c'est-à-dire des coûts marginaux ».

En ce qui concerne les conditions de travail, l'étude Input (« Libéralisation et précarisation ») établit une comparaison avec DPAG dans trois domaines : les types de contrats, "les situations de revenus » et la représentation des intérêts des salariés au sein des entreprises concurrentes. Et, conformément à l'approche que révèle son titre, elle se concentre sur l'emploi atypique. S'appuyant sur les statistiques de l'autorité de régulation, elle rappelle ainsi que ces petits boulots représentent $62,3 \%$ des emplois dans le secteur concurrentiel (ils ne sont que $1 \%$ à DPAG), soulignant que, dans aucune autre branche intensive en main d'œuvre (propreté : $56,3 \%$ ou gastronomie : 52,8\%), ceux-ci «n'ont atteint de telles proportions ». Et d'en conclure à «une insécurité caractérisée de l'emploi » dans ce secteur, quelques lignes seulement après avoir expliqué que, dans cette catégorie, « retraités et chômeurs bénéficiaires de l'Arbeitslosengeld I et II [respectivement allocations et revenu forfaitaire d'insertion; IB] représentent au total plus de $40 \%$ des salariés des nouveaux prestataires ». A quoi il faut ajouter un certain nombre d'étudiants. Mais cette catégorie, bien qu'évoquée plus loin au détour d'une phrase, n'est pas chiffrée ; pourtant, elle constitue le pilier du portage des quotidiens - un job étudiant classique outre-Rhin. De même, dans un tout autre contexte (faibles qualifications), sont évoquées les femmes. Autrement dit, la moitié au moins de ces petits boulots sont occupés pour générer des revenus complémentaires (au salaire du mari, aux allocations, pensions ou aux prêts étudiants versés au titre du Bafög) ou pour ménager une réinsertion dans la vie professionnelle aux chômeurs de longue durée (cumul sous conditions avec l'indemnité 'Hartz IV' promu par la loi).
Une étude de la Bundesnetzagentur est en suspens

L'étude Input commanditée par Ver.di se focalise sur la précarisation de l'emploi...

... sur un marché où le seul avantage concurrentiel est le prix

Focus sur les petits boulots : précarisation ou source complémentaire de revenus ? 
Etude Input : un plaidoyer pour une régulation sociale du marché

Un seul instrument possible : le salaire minimum

Promouvoir un nouveau mode de régulation salariale par la loi

Des revendications récurrentes de ver.di : pour un SMIC légal...
Pour résumer : le propos de l'étude est de démontrer la précarité de l'emploi dans ce secteur à bas salaires, de surcroît non organisé. Elle le fait dans les règles de l'art de la persuasion, offrant une lecture orientée de chiffres incontestables, recourant à un discours circulaire et émaillant l'analyse d'interviews illustrant le climat délétère qui règne dans ces entreprises gouvernées par une " hiérarchie autoritaire ». La conclusion est simple: «les implications négatives de l'emploi précaire révèlent la nécessité de mesures adaptées dans le sens d'une 'politique de la déprécarisation' ». Cinq options sont proposées : l'abandon ou le ralentissement de l'ouverture au marché ; l'accompagnement de la libéralisation par une mise en œuvre plus sévère des "standards de protection sociaux » de la Loi Postgesetz; le recours généralisé à une clause de 'mieux disant social' (Tariftreueklausel) ; la conclusion d'une convention collective pour la distribution de la lettre ; l'institution d'un salaire minimum sectoriel ou généralisé.

La République fédérale s'étant engagée à ouvrir le marché postal dans les délais et à tenir ses engagements européens, il ne restait qu'à poursuivre la sensibilisation de l'opinion sur une éventuelle régulation sociale. En procédant en quelque sorte par élimination. Une interprétation plus 'poussée' de la «clause sociale » la Loi Postgesetz, une disposition qui n'existe dans aucun autre texte allemand libéralisant un ancien service public, est délicate du point de vue juridique : d'une part, elle est incompatible avec le rôle de gardien de la concurrence imparti à titre transitoire à la Bundesnetzagentur et, plus généralement, à l'Office fédéral des Cartels ; d'autre part, elle impliquerait une ingérence du législateur dans l'autonomie des partenaires sociaux en violation des principes constitutionnels allemands; enfin, elle constituerait une barrière à la libre circulation des services en contradiction avec la Directive services qui entrera en vigueur le 01-01-2009. L'application généralisée de clauses de 'mieux disant social' au marché postal (elles existent dans le BTP) serait peu conforme à la liberté d'établissement garantie par le droit national et européen, indépendamment du fait que cette possibilité est réservée aux procédures de passation de marchés publics. Quant à la signature d'une convention tarifaire, elle se heurte à la non organisation de la branche et l'émiettement des partenaires sociaux. Restait l'institution d'un salaire minimum sectoriel.

Normalement, les seuils salariaux, à commencer par les planchers, se définissent outre-Rhin selon une logique contractuelle, au niveau de la branche et non de métiers. Mais ces 'nouveaux' services postaux ne répondent pas à la définition de la branche. Et encore moins le métier de facteur, au centre de toutes les préoccupations. Par ailleurs, l'idée même de l'instauration d'un salaire minimum légal, ne serait-ce qu'au niveau d'une branche, est contraire à la garantie constitutionnelle de l'autonomie tarifaire - sauf lorsqu'une telle disposition est justifiée par un intérêt supérieur. Restait donc à ver.di comme aux partis de gauche, puis au SPD membre de la grande coalition fédérale, de créer les conditions pour que s'impose à l'évidence un impératif d'intérêt supérieur.

Ils y sont parvenus grâce à une stratégie de longue haleine, s'étendant sur plusieurs années, et dont l'objectif final est la généralisation de la fixation des salaires par la loi dans la totalité du secteur des services. Dans ce contexte, le SMIC postal ne constitue dès lors qu'une première étape. Cette stratégie, qui est très étrangère à la culture de négociation sociale allemande telle qu'elle caractérise toujours le secteur de l'industrie où œuvrent des syndicats unitaires établis comme IG Metall ou IG BCE, repose, pour le nouveau syndicalisme du tertiaire, sur une maîtrise professionnelle de la communication politique et de l'agenda setting dans l'espace public. Cette stratégie est propre à ver.di, un conglomérat de métiers de services (du chanteur d'opéra à l'éboueur), né en 2001 de la fusion de cinq organisations et qui, faute d'une culture syndicale unitaire à l'image d'un IG Metall, s'est fait fort de développer une fonction tribunicienne pour construire sa légitimité. Elle suit une "logique d'actions ponctuelles et très ciblées, mettant à profit les lois propres à l'information » (Pautrat, 2005) et recourt fréquemment aux possibilités offertes par Internet, par exemple, pour mener des actions de type corporate campaigns à l'encontre de certaines entreprises (celle contre le discounter Lidl fait figure de cas 
d'école). Frank Bsirske, président du syndicat et membre du parti des Verts, avait présenté ainsi la stratégie de son organisation dans une interview accordée à la presse syndicale (Gewerkschaftliche Monatshefte, 9/2004): "plus Greenpeace que puissance gréviste ». Cette approche se manifeste principalement via deux chevaux de bataille - autant de thèmes fédérateurs pour une organisation en mal de cohésion : la revendication d'une approche distributive de l'Etat social, et la défense des services d'intérêt général (dénommés Daseinsvorsorge en allemand).

Or ce faisant, ver.di quitte le rôle traditionnel de partenaire social en charge de la défense des intérêts des salariés de sa branche et se positionne en tant que mouvement d'opinion sur l'échiquier politique où, pour mieux faire entendre sa voix, il cultive les partenariats à gauche du SPD. Ainsi, à l'été 2004, soit six mois avant l'entrée en vigueur de la Loi « Hartz IV » qui instaure une sorte de RMI sur la base d'un forfait mensuel de $345 €$ (voir REA 68/04) pour inciter les chômeurs au retour en emploi, ver.di s'était allié avec le mouvement ATTAC pour manifester contre ce "démantèlement » de l'Etat social. La programmation régulière de ces manifestations (tous les lundis), en pleine campagne électorale pour les législatives dans le Land de Brandebourg, garantissait l'attention médiatique. Auparavant, ver.di avait durci ses positions face à un SPD accusé lui aussi, sous la direction du chancelier Schröder, de trahir l'Etat social avec son Agenda 2010. C'est sous le gouvernement Schröder II que, pour ouvrir un segment à bas salaires indispensables à la lutte contre le chômage de longue durée des moins qualifiés, avait été assoupli le régime des petits boulots (dont les mini-jobs) - ce même régime qu'avait réglementé de manière très restrictive le gouvernement Schröder I...

Mais entre-temps, et bien que les deux gouvernements fédéraux successifs aient été une coalition SPD/Verts, le chancelier avait 'recentré' sa politique. Or l'Agenda 2010 avait divisé irrémédiablement le SPD, entraînant la démission de G. Schröder de la direction de son parti, puis la programmation d'élections anticipées au Bundestag dont est issu à l'automne 2005 l'actuel gouvernement de grande coalition mené par A. Merkel (CDU). Depuis 2007, à l'approche d'une série d'échéances électorales importantes culminant dans les élections au Bundestag à l'automne 2009, le SPD, traditionnellement un parti de centre-gauche, s'est repositionné sur sa gauche, en réaction aussi à la montée en puissance d'une extrême-gauche menée par le nouveau parti Die Linke qui réunit des transfuges du SPD (Oskar Lafontaine) et le PDS, héritier du parti communiste de l'ex-RDA. Ce 'gauchissement' progressif d'une partie du champ politique allemand crée un contexte favorable pour le retour en arrière sur un certain nombre de réformes sociales de l'Agenda 2010, de même que pour la montée en puissance de revendications redistributives. Le retour de la croissance contribue de son côté à les nourrir.

En 2007, le contexte est donc propice pour convaincre le ministre fédéral du Travail (SPD) puis le gouvernement fédéral de la nécessité de réglementer par la loi, sous couvert de la protection des droits sociaux, un service d'intérêt général : le marché postal émergent. Le constat de défaillance des partenaires sociaux constitue un argument pro domo imparable. Le message passe d'autant mieux dans l'opinion que l'accent est mis, dans le discours syndical ou politique, sur la destruction d'emplois liée à la privatisation, la tendance à la précarisation et la baisse générale du pouvoir d'achat. «Un accompagnement social » de l'ouverture des marchés postaux s'impose donc pour "enrayer la menace de voir dériver le marché de la lettre vers une zone de pauvreté et de salaires de famine ", explique ainsi ver.di dans un plaidoyer pour le SMIC postal publié à l'automne 2007 et intitulé : «Pour un salaire dont on puisse vivre ! » (Für einen Lohn, von dem man leben kann !). Et, à l'appui de ses revendications, il reproduit le chiffre clef provenant de l'édition d'octobre du baromètre mensuel réalisé par l'institut Infratest-dimap pour la chaîne publique $\mathrm{ARD}$, un baromètre réputé pour son sérieux : $86 \%$ des sondés au total se sont déclarés « favorables à l'instauration d'un salaire minimum dans certaines branches » (27\%) ou «à son instauration générale » (59\%). Dans un contexte économique global de pouvoir d'achat en stagnation, et alors que la question d'un salaire minimum figure en tête de l'agenda politique depuis plusieurs mois, une formulation aussi générale de la question
... contre les minijobs et la loi « Hartz IV »

Un contexte politique favorable à une logique de redistribution

Le SPD s'empare de l'idée d'un SMIC légal 
(pour/contre) ne peut que se solder par une majorité de réponses favorables, y compris sur la base d'un panel représentatif. Or ce même sondage, ramené dans ce cas aussi à cet unique pourcentage, a constitué l'un des arguments clefs de la campagne du SPD lors des législatives dans le Land de Hesse à la fin janvier 2008. C'est à cette occasion aussi que s'est confirmée l'alliance, autour du thème de l'introduction en Allemagne d'un SMIC légal généralisé, entre SPD, Die Linke, ver.di et les Verts.

\begin{tabular}{|c|}
\hline Par quels biais a été instauré le salaire minimum légal pour le marché postal \\
\hline $\begin{array}{l}\text { La législation allemande prévoit trois cas de figure où l'intérêt général justifie une entorse à l'autonomie tarifaire des partenaires } \\
\text { sociaux garantie par la Loi fondamentale: }\end{array}$ \\
\hline $\begin{array}{l}\text { - le recours à la Loi sur les conditions de travail minimales (Mindestarbeitsbedingungsgesetz, Mia) du 11-01-1952, qui permet au } \\
\text { législateur de se substituer à des partenaires sociaux défaillants ; mais elle n'a jamais été appliquée ; }\end{array}$ \\
\hline 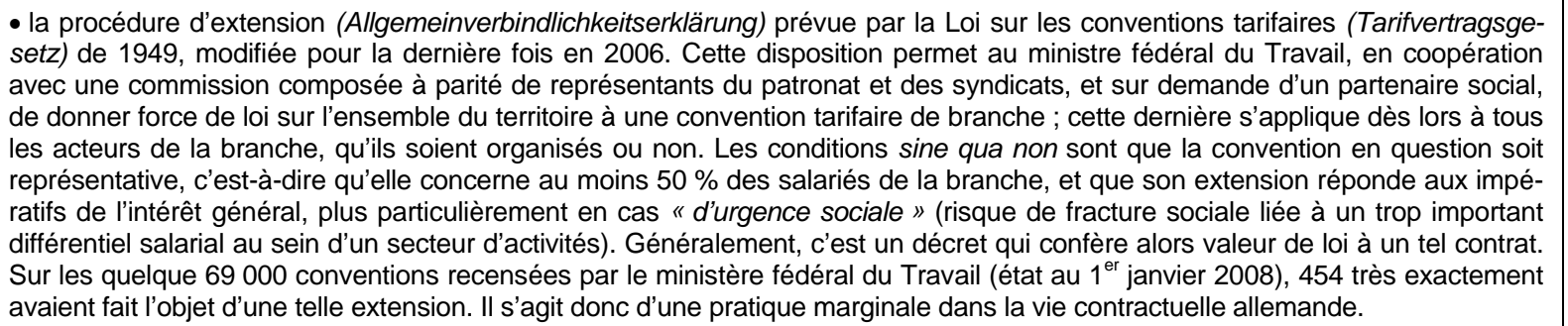 \\
\hline $\begin{array}{l}\text { - Une troisième possibilité a été introduite plus récemment, par l'adoption de la Loi Arbeitnehmer-Entsendegesetz (AEntG) du } 26 \text { - } \\
\text { 02-1996, votée en prévision de l'adoption de la Directive 71/96/CE sur les travailleurs détachés (un texte fondé sur le principe du } \\
\text { pays d'accueil contrairement à la Directive Services). Si son objectif officiel est la lutte contre le travail illégal, son but véritable est } \\
\text { d'écarter le risque de “dumping salarial » émanant d'entrepreneurs proposant " des prix défiant toute concurrence ", comme } \\
\text { l'expliquaient alors sur leur site les Douanes allemandes, chargées d'en contrôler l'application (voir REA 71/05). La Loi } A E n t G \\
\text { visait à protéger les principales catégories de salariés du BTP est-allemand contre les concurrents est-européens grâce à des } \\
\text { salaires minima d'au moins } 7 \text { €/h (peintre formé sur le tas). Le domaine d'application de la loi a été étendu à l'automne } 2006 \text { à } \\
\text { d'autres métiers du BTP. C'est le seul cas où ait été instauré un SMIC légal en Allemagne en dix ans. }\end{array}$ \\
\hline 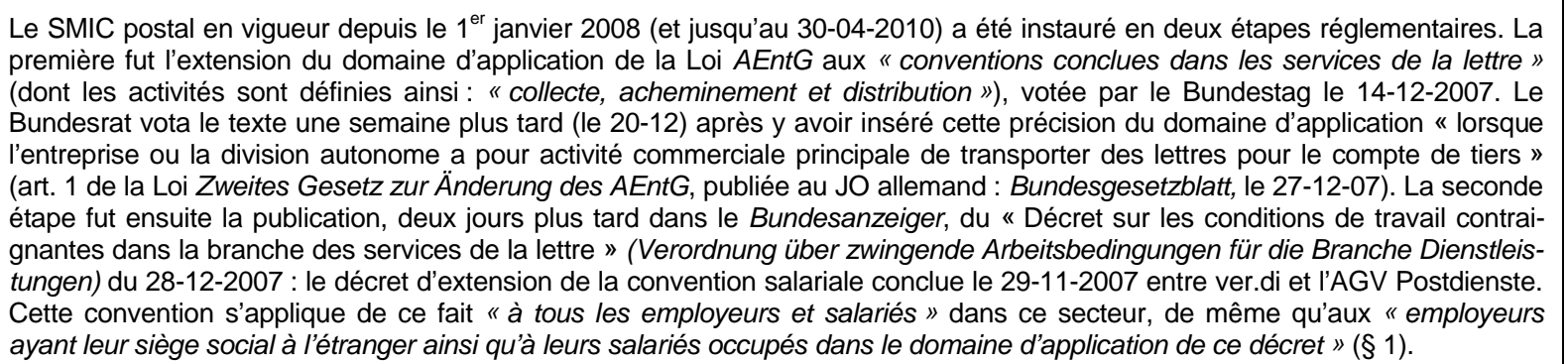 \\
\hline
\end{tabular}

L'idée d'un SMIC en masque une autre : la hausse de tous les salaires dans les services
Le cas de l'ouverture du marché postal a ainsi permis à ver.di non seulement de rouvrir le dossier, provisoirement clos en 1996 par l'adoption d'une « clause sociale » et rouvert lors de l'entrée en vigueur de la loi « Hartz IV » puis refermé du fait de l'opposition des autres syndicats. II a aussi permis de remettre à l'ordre du jour dans un contexte plus porteur (SPD et coalition fédérale plaident désormais pour un plafonnement des salaires patronaux ; l'affaire de la fraude fiscale impliquant l'ex-PDG de DPAG, Klaus Zumwinkel, et révélée à la mi-février 2008, tombe pour ainsi dire à point nommé) une revendication fondamentale pour le syndicat: une hausse générale des salaires dans le secteur des services. Car c'est de cela qu'il s'agit en réalité. Déjà, au printemps 2005, lors de la remise en débat du projet de Directive Services, le gouvernement fédéral SPD/Verts s'était emparé des revendications de ver.di - en pleine campagne électorale pour les législatives de Rhénanie du Nord-Westphalie, à l'approche des élections au Bundestag et sur fond de tensions au sein du SPD : en avril, son secrétaire général, Franz Müntefering, déclare la guerre au « capitalisme » lors de son discours ouvrant le débat sur la refonte du programme du SPD (adopté à l'automne 2007, voir REA 84/07). La coalition fédérale prônait alors, pour contrer le « dumping salarial » que favoriserait cette directive, l'adoption d'un SMIC légal comme la généralisation des dispositions sur les travailleurs détachés contenues dans la loi afférente de 1996. II ne restait plus qu'à trouver le moment opportun pour nouer ces fils et déboucher sur l'adoption d'un SMIC légal dans les services postaux - avec 
un salaire plancher nettement supérieur à la fois aux usages en vigueur dans le secteur des services et au salaire minimum implicite de référence que constitue l'aide sociale. Et pour 'détricoter' ainsi, en passant, les lois Hartz qui, notamment en abaissant les seuils des revenus de substitution, cherchaient à inciter au retour à l'emploi les chômeurs les moins qualifiés. II est vrai que c'est dans le tertiaire que se trouvent les plus faibles rémunérations dans l'échelle allemande plus précisément, les plus faibles planchers salariaux, fixés, soit dit en passant, par ver.di lui-même (voir REA 71/05).

C'est ainsi que, d'une surenchère électoraliste à l'autre, la CDU/CSU a été prise de court. Certes, le ministre fédéral de l'Economie (CSU) proclamait à l'automne qu'il ne céderait pas sur ce point, considérant, tout comme la chancelière (CDU) que l'instauration d'un SMIC dans les activités intensives en main d'œuvre, non content de réduire l'emploi, équivaudrait à un retour en arrière dans la politique de levée des obstacles à l'embauche. Mais le gouvernement de grande coalition a fini par légiférer, une fois la présidence allemande de l'UE passée. Le moment choisi n'est pas innocent non plus sous l'angle de la réglementation communautaire, puisque c'est via la loi sur les travailleurs détachés qu'a été introduit le SMIC postal. En effet, "les caractéristiques structurelles des services postaux, en liaison avec la libéralisation des marchés postaux à l'échelle européenne, exigent, dans ces activités aussi, le recours au dispositif de [cette] loi ». (attendus de l'art. 1 de la Loi $A E n t G$ modifiée). A l'approche de cette libéralisation comme de l'entrée en vigueur de la Directive services, la protection « sociale » des préposés permet aussi d'ériger de nouvelles barrières protectionnistes tout en respectant par ailleurs l'échéancier européen...

Ce SMIC vise en réalité un « objectif de politique industrielle : préserver le monopole de la lettre », critique le président du Conseil des Sages (Handelsblatt, 0512-07), abondant dans le même sens que la Commission des monopoles dont le rapport sur le marché postal s'intitule: «Une lutte par tous les moyens pour la survie du monopole ». Car les mêmes barrières s'élèvent à l'entrée des concurrents nationaux, le SMIC ayant été fixé en référence implicite aux tarifs spéciaux en vigueur chez DPAG, soit à un niveau sans commune mesure avec les impératifs économiques d'un marché émergent intensif en main d'œuvre faiblement qualifiée et où le facteur salaire joue un rôle décisif. Face à ce constat, PIN AG a engagé à la fin décembre une procédure d'insolvabilité ; TNT Post et Hermes ont réduit leurs investissements dans le réseau allemand, le premier allant jusqu'à envisager son retrait d'Allemagne. Car à la problématique salariale s'ajoute l'exonération de la TVA dont bénéficie DPAG en vertu du décret sur le service universel. Ce texte est certes actuellement en révision, mais vise à définir les obligations de service universel de telle manière (couverture de l'ensemble du territoire) que seule la DPAG peut les remplir, surtout si les intégrateurs concurrents déclarent forfait. La Fédération BdKEP a donc saisi en janvier la Direction fiscalité et Union douanière de la Commission européenne. Cette saisine s'ajoute à une procédure d'infraction engagée par la Commission en 2007 contre l'Allemagne sur le mode de réglementation des prix de DPAG, jugé peu transparent et s'assimilant lui aussi à une aide d'Etat (avec la connivence de l'autorité de régulation, comme le rappelle la Commission des monopoles).

Mais on peut se demander si le gouvernement fédéral a réellement intérêt à lever le monopole de DPAG, sachant qu'il a placé ses parts de capital $(30,6 \%)$ auprès de la banque publique KfW, chargée de les mettre sur le marché pour achever la privatisation de DPAG. Or la plus-value réalisée revenant au Bund, celui-ci a tout à gagner à préserver le plus longtemps possible la rente de monopole de son opérateur pour en accroître la valeur boursière, rappelle la Commission des monopoles.

VISIBLEMENT, L'ALLEMAGNE EPROUVE DE GRANDES DIFFICULTES A LIBERALISER SES INDUSTRIES DE RESEAUX, comme le montrent aussi les exemples de l'énergie ou des télécommunications (voir REA 76/06 et 78/06). Elle est loin d'être le seul parmi les Etats membres, ce dont attestent la multitude de procédures en cours à
Une mesure protectionniste au nom de la justice sociale

Pour le gouvernement, un moyen de proroger le monopole...

... en attendant de réaliser une plus-value boursière 
la Commission, les atermoiements et affrontements dans le secteur énergétique, le débat européen sur la régulation des industries de réseaux, ou les débats en cours sur la définition des services d'intérêt général. Mais l'Allemagne innove en re-régulant son marché postal, à peine ouvert, via la réglementation des salaires. $\mathrm{Et}$, ironie de l'histoire, en le faisant le jour même du $50^{\mathrm{e}}$ anniversaire de l'Office fédéral des Cartels et de la Loi contre les entraves à la concurrence, balayant d'un décret à visée purement électoraliste un demi siècle de doctrine anti-cartels !

Certes, la juridiction allemande aura à connaître de cette violation de l'autonomie constitutionnelle des partenaires sociaux, et peut-être la recommandation de la Commission des monopoles sera-t-elle suivie d'effet: à savoir de soumettre à l'avenir à l'avis de l'Office fédéral des Cartels tout projet de procédure d'extension d'une convention salariale. Ce serait en tout cas une réponse adaptée à la nouvelle donne introduite dans l'approche de la concurrence dans le tertiaire, où le droit social tend à s'ériger en instrument parallèle de régulation des marchés. Car les SMIC « ont tendance à devenir politiquement incontrôlables " (Elsenbast, 2007). Certes, pour l'instant ne s'esquisse qu'une dualisation de la régulation sociale allemande (voir REA 83/07) : l'industrie et le tertiaire ouvert à la concurrence, comme les services financiers, recourent à la voie établie du contrat dont les normes s'orientent en fonction du marché et de la compétitivité ; le tertiaire en voie de dérégulation en appelle à la loi au nom d'un ordre public social. Mais la tendance à légiférer sur les salaires pourrait s'étendre aux branches organisées. Le ministère fédéral du Travail (SPD), en tout cas, s'apprête à réviser la loi de 1952 sur les conditions de travail minimales en coopération avec le DGB pour y aménager cette possibilité au nom d'un "intérêt général supérieur ": la baisse des transferts sociaux (subventions salariales, voir REA 83/07). "Adieu, autonomie tarifaire!", s'exclame un éditorial du quotidien Frankfurter Allgemeine Zeitung (15-06-2007). A propos du dossier postal, avec la complicité du SPD qui en fut l'artisan historique, et au nom de la lutte contre la précarité, l'Allemagne est tout simplement en train de « démanteler » les principes fondateurs de son modèle social...

\section{Indications bibliographiques}

BAUER T, SCHMIDT C., " Mindestlöhne und das Entsendegesetz », Wirtschaftsdienst, n`3/07 BOURGEOIS I., " Directive 'Services' : les enjeux du débat allemand » et « La difficile émergence d'un marché de la lettre en RFA », Regards sur l'économie allemande, $n^{\circ 5} 71 / 05$ et 65/04, et " La libéralisation du marché postal allemand : entre service public et marché ", in BouRGEOIS I. (dir.), Allemagne 2001, Regards sur une économie en mutation, Editions du CIRAC, 2001

Brandt T., Drews K., Schulten T., "Liberalisierung des deutschen Postsektors - Auswirkungen auf Beschäftigung und Tarifpolitik », WSI Mittelungen, $n^{\circ} 5 / 2007$

BRENKE K, EICHHORST W., « Mindestlohn für Deutschland nicht sinnvoll » DIW-Wochenbericht, $n^{\circ} 9 / 2007$

"Des relations sociales en plein bouleversement », Un entretien avec le Prof. Wolfgang Schrœder, Regards sur l'économie allemande, $n^{\circ} 83 / 07$

DIEKE Alex K., ZAUNER M., Arbeitsbedingungen im Briefmarkt, Wissenschaftliches Institut für Infrastruktur und Kommunikationsdienste (WIK), mai 2007, www.wik.org

ELSENBAST W., « Mindestlöhne im Postsektor? ", Wirtschaftsdienst, $n^{\circ} 4 / 07$

Liberalisierung und Prekarisierung - Beschäftigungsbedingungen bei den neuen Briefdienstleistern in Deutschland, Input Consulting, décembre 2006, www.input-consulting.de

PaUtrat M-H., "Privatisation et dérégulation en RFA : les limites du pragmatisme syndical ", Regards sur l'économie allemande, n58/2002, et « Ver.di : un géant sans cohésion ", in BOURGEOIS I. (dir.), Le modèle social allemand en mutation, Editions du CIRAC, 2005

SÄCKER F. J., Soziale Schutzstandards im Postregulierungsrecht, Rechtsgutachten erstattet der Bundesnetzagentur, janvier 2007

SCHRCEDER W., " Das Band der Solidarität neu knüpfen ? ", Mitbestimmung, $n^{\circ} 5 / 07$

Tätigkeitsbericht 2006/2007 der Bundesnetzagentur. Lage und Entwicklung auf dem Gebiet des Postwesens, décembre 2007, www.bundesnetzagentur.de

Telekommunikation und Post 2003: Wettbewerbsintensivierung in der Telekommunikation Zementierung des Postmonopols. Sondergutachten der Monopolkommission, décembre 2003, www.monopolkommission.de

Wettbewerbsentwicklung bei der Post 2007: Monopolkampf mit allen Mitteln. Sondergutachten der Monopolkommission, décembre 2007, www.monopolkommission.de

Zehnte Marktuntersuchung für den Bereich der lizenzpflichtigen Postdienstleistungen, fin 2006, Bundesnetzagentur, Referat 312, www.bundesnetzagentur.de 